\title{
Zur geologischen Altersbestimmung nach der Kalium々Argon»Methode
}

\author{
Von W. Gentner und W. Kley \\ Aus dem Physikalischen Institut der Universität Freiburg (Brg.) \\ (Z. Naturforschg. 10 a, 832-833 [1955]; eingegangen am 23. August 1955) \\ J. Mat tauch zum 60. Geburtstag gewidmet
}

$\mathrm{I}^{\mathrm{n}}$

n unseren früheren Arbeiten ${ }^{1-4}$ hatten wir bereits gezeigt, daß durch den dualen Zerfall des $\mathrm{K}^{40} \stackrel{\beta}{\rightarrow} \mathrm{Ca}^{40}$ und $\mathrm{K}^{40} \stackrel{\mathrm{K}}{\rightarrow} \mathrm{A}^{40}$ die Möglichkeit besteht, den letzgenannten Übergang zur geologischen Altersbestimmung heranzuziehen.

Die exakte Altersbestimmung setzt die genaue Kenntnis der Zerfallskonstanten des $\mathrm{K}^{40}$ und die vollständige Gewinnung des gebildeten Argon voraus. Es kann heute mit Sicherheit angenommen werden, das jeder K-Übergang des $\mathrm{K}^{40}$ mit einem $\gamma$-Quant gekoppelt ist, und deshalb die Zahl der $\gamma$-Quanten pro Gramm Kalium und Sekunde direkt die Zerfallskonstante für den K-Übergang liefert. Neuere Messungen ${ }^{5-10}$ ergaben für die Zerfallsrate 3,0 bis 3,6 K-Übergänge pro Gramm Kalium und Sekunde. Für unsere Alterswerte benutzten wir daher einen mittleren Wert von $(3,3 \pm 0,3) \gamma / \mathrm{g} \mathrm{Ka}$ lium $\cdot$ sec und ein Verzweigungsverhältnis von $\lambda_{\mathrm{K}}$ : $\lambda_{\beta}=0,119$, das wir in der vorliegenden Arbeit weitgehend bestätigen konnten.

In Fortsetzung unserer Altersbestimmungen an tertiären Kalisalzen ( $\mathrm{G}$ e n tn e r, S mits) wurden neuerdings von uns auch Argonmessungen an Kalifeldspäten durchgeführt. Zur Gewinnung des Argon aus dem Feldspat haben wir zwei Methoden angewandt :

1. Aufschluß des Materials in einer $\mathrm{Na}_{2} \mathrm{O}_{2}$ Schmelze und

2. Schmelzen der Probe im Hochfrequenzofen.

Die Reinigung und Bestimmung des Argon erfolgte im wesentlichen nach der von $G$ e $n t n$ e $r$ und $\mathrm{S} \mathrm{m}$ it s beschriebenen Methode. Als Untersuchungsmaterial wurde ein Mikroklin aus dem Pegmatit von Varuträsk (Nordschweden) verwendet. Es stand ein Stück von ungefähr $300 \mathrm{Gramm}$ zur Verfügung, das

\footnotetext{
1 F. S m it s u. W. G e n t ner, Geochim. cosmochim. Acta (London) 1, 22 [1951].

${ }_{2}$ W. Gentner, R. Präg u. F. Smits, Geochim. cosmochim. Acta (London) 4, 11 [1953].

${ }_{3}$ W. G e n t n e r, K. G o e b e l u. R. Präg, Geochim. cosmochim. Acta (London) 5, 124 [1954].

${ }^{4}$ W. G entner, F. Jensen u. K. R. Mehnert, Z. Naturforschg. 9a, 176 [1954].
}

rein äußerlich wie ein Einkristall aussah, und von dem jeweils ein Stück von 2 bis 5 Gramm unzerkleinert für eine Messung verwendet wurde. Die Bestimmung des Kaliumgehaltes wurde flammenphotometrisch durchgeführt, und der Luftargongehalt der Probe massenspektrometrisch aus dem Isotopenverhältnis $\mathrm{A}^{40}: \mathrm{A}^{36}$ ermittelt. Er lag bei den meisten Proben unter 15\%. In Tab. 1 sind zunächst die Meßergebnisse aus der $\mathrm{Na}_{2} \mathrm{O}_{2}$-Aufschlußmethode zusammengestellt.

\begin{tabular}{|c|c|c|c|c|c|}
\hline Material & Nr. & $\%$ Kal. & $\frac{\mathrm{mm}^{3} \mathrm{rad} . \mathrm{A}^{40}}{\mathrm{~g} \text { Material }}$ & $\frac{\mathrm{mm}^{3} \text { rad. } A^{40}}{\mathrm{~g} \mathrm{Kalium}}$ & Alter \\
\hline $\begin{array}{l}\text { Mikroklin } \\
\text { Varuträsk }\end{array}$ & $\begin{array}{l}415 \\
416 \\
417 \\
418 \\
419 \\
420 \\
423\end{array}$ & $\begin{array}{l}10,89 \pm 0,08 \\
11,00 \\
11,00 \\
11,00 \\
10,96 \\
11,08 \\
11,00\end{array}$ & $\begin{array}{l}0,874 \\
0,882 \\
0,862 \\
0,932 \\
0,695 \\
0,685 \\
0,900\end{array}$ & $\begin{array}{l}8,03 \pm 5 \% \\
8,03 \\
7,84 \\
8,47 \\
6,35 \\
6,20 \\
8,18\end{array}$ & $\begin{array}{l}1,40 \cdot 10^{9} \mathrm{a} \\
1,40 \\
1,37 \\
1,45 \\
1,18 \\
1,16 \\
1,42\end{array}$ \\
\hline
\end{tabular}

Tab. 1. Ergebnisse der Argongewinnung durch $\mathrm{Na}_{2} \mathrm{O}_{2}$-Aufschluß.

Bei dieser Methode der Argongewinnung kann der Kaliumgehalt nicht direkt an den Proben selbst bestimmt werden, sondern nur an einem benachbarten Stück. Dadurch können erhebliche Fehler verursacht werden. Um diese möglichen Fehler zu kontrollieren, wurde die Argongewinnung noch nach einer zweiten Methode durchgeführt.

Bei dem zweiten Aufschlußverfahren wurde die Probe mit einem HF-Generator von $6 \mathrm{~kW}$ Leistung in einem Graphittiegel bei einer Temperatur von $1700^{\circ} \mathrm{C}$ geschmolzen. Dadurch war es möglich, die Kaliumanalyse am Schmelzgut selbst auszuführen. Dies erscheint uns besonders wichtig bei der Argongewinnung an Graniten, die in kleinen Bereichen bereits Schwankungen des Kaliumgehaltes bis zu 30\% aufweisen.

5 G. A. Sawyer u. M. L. Wied enbeck, Phys. Rev. 76, 1535 [1949]; 79, 490 [1950].

${ }^{6}$ F. G. Hout ermans, O. H a x el u. F. Heintze Z. Phys. 128, 657 [1950].

7 W. R. Fa u st , Phys. Rev. 78, 624 [1950].

8 M. L. G o od, Phys. Rev. 81, 891 [1951]; 83, 1054 [1951].

9 P. R. J. B u r c h, Nature, Lond. 172, 361 [1953].

10 R. W. S t o u t, Phys. Rev. 75, 1107 [1949]. 
Durch Testversuche konnte außerdem gezeigt werden, daß auch bei einer Temperatur von $1700^{\circ} \mathrm{C}$ aus der $\mathrm{SiO}_{2}$-reichen Schmelze kein Kalium abdampft. In Tab. 2 haben wir die Meßergebnisse der Argongewinnung von 7 Proben nach dem Schmelzverfahren im HF-Ofen zusammengestellt.

\begin{tabular}{|c|c|c|c|c|c|}
\hline Material & Nr. & $\%$ Kal. & $\frac{\mathrm{mm}^{3} \mathrm{rad} . \mathrm{A}^{4 \mathrm{C}}}{\mathrm{g} \text { Material }}$ & $\frac{\mathrm{mm}^{3} \mathrm{rad} . \mathrm{A}^{40}}{\mathrm{~g} \text { Kalium }}$ & Alter \\
\hline $\begin{array}{l}\text { Mikroklin } \\
\text { Varuträsk }\end{array}$ & $\begin{array}{r}509 \\
510 \\
511 \\
512 \\
513 \\
514 \\
29\end{array}$ & $\begin{array}{l}11,00 \pm 0,08 \\
11,00 \\
11,00 \\
11,00 \\
11,00 \\
11,00 \\
11,00\end{array}$ & $\begin{array}{l}0,947 \\
0,803 \\
0,743 \\
0,931 \\
0,730 \\
0,791 \\
0,886\end{array}$ & $\begin{array}{l}8,62 \pm 5 \% \\
7,31 \\
6,76 \\
7,56 \\
6,64 \\
7,19 \\
8,05\end{array}$ & $\begin{array}{l}1,47 \cdot 10^{9} \mathrm{a} \\
1,31 \\
1,235 \\
1,34 \\
1,2 \cdot 2 \\
1,29 \\
1,40\end{array}$ \\
\hline
\end{tabular}

Tab. 2. Ergebnisse der Argongewinnung im HF-Ofen.

Vergleicht man Tab. 1 und 2, so ersieht man daraus, daß der Argongehalt pro Gramm Kalium bei beiden Aufschlußmethoden sich beträchtlich ändern kann, nämlich von 6,20 bis $8,62 \mathrm{~mm}^{3}$ rad. $\mathrm{A}^{40} / \mathrm{g}$ Kalium. Dieser unterschiedliche Argongehalt desselben Materials kann keineswegs auf eine Unvollständigkeit der Argongewinnung zurückgeführt werden. Mehrere Testversuche zeigten nämlich bei beiden Methoden, daß durch wiederholtes AufschlieBen oder Schmelzen kein Argon mehr gefunden werden kann. Die verschiedenen Argonwerte liegen auch weit außerhalb der Fehlergrenzen der Argon-KaliumBestimmung, die mit einer Genauigkeit von $4-5 \%$ ausgeführt werden kann. Die beträchtlichen Schwankungen im Argongehalt können also nur auf wirkliche Argonverluste im Material zurückgeführt werden. Zur Aufklärung dieser Tatsache wurden zehn Dünnschliffe des Mikroklin angefertigt und mikroskopisch untersucht. Dabei zeigte sich, daß das Material stellenweise stark kaolinisiert war. Diese Kaolinisierung muß natürlich zu Argonverlusten führen und bedingt offenbar den verschiedenen Argongehalt. Inwieweit auch allgemeine Diffusionsvorgänge am Argonverlust beteiligt sind, konnten wir bis jetzt noch nicht aufklären.

Vor kurzem wurde auch von $\mathrm{Reker}^{11}$ eine

11 H. R e k e r, Dipl.-Arbeit, T. H. München.

12 Für die Halbwertszeit des Rb wurde der Wert $6,27 \cdot 10^{10}$ a benutzt.

13 Selbst unter Heranziehung der älteren Altersbestimmungen von Varuträsk von W. W a h l (in P. Quensel, Geol. Fören. Förh. 62, 395 [1940] : 1,70 $10^{9} \mathrm{a}$ und S. Eklund (Ark. Math., Astron. Fys. 33 a, No. 14 [1946]) : $(1,7 \pm 0,2) \quad 10^{9} \mathrm{a}$ ergibt sich bei einem Mittelwert von $1,65 \cdot 10^{9} \mathrm{a}$ für $\lambda_{\mathrm{K}}: \lambda_{\beta}$ $=0,099$.
Altersbestimmung des Pegmatits aus Varuträsk nach der $\mathrm{Rb} / \mathrm{Sr}$-Methode durchgeführt. Er bestimmte spektralanalytisch das $\mathrm{Rb} / \mathrm{Sr}$-Verhältnis mit einer Genauigkeit von $\pm 2,5 \%$ und konnte mit Hilfe des von $\mathrm{M} \mathrm{at} \mathrm{t}$ a u $\mathrm{ch}$ bestimmten Isotopenverhältnisses $\mathrm{Sr}^{87} / \mathrm{Sr}^{88}$ für den Pegmatit das Alter von (1,59 \pm $0,08) \cdot 10^{9}$ a angeben ${ }^{12}$. Mit diesem Sr-Alter und dem von uns gemessenen maximalen Argongehalt des Mikroklin von $8,62 \mathrm{~mm}^{3} \mathrm{rad} . \mathrm{A}^{40} / \mathrm{g}$ Kalium können wir das Verzweigungsverhältnis des $\mathrm{K}^{40}$ berechnen. Wir erhalten ${ }^{13}: \lambda_{\mathrm{K}}: \lambda_{\beta}=0,104$. Dieser Wert kann wegen der oben diskutierten Argonverluste nur als eine untere Grenze für das Verzweigungsverhältnis betrachtet werden. In diesem Zusammenhang wären auch die Arbeiten von Wasserburg ${ }^{14,15}$ und R u s s e ${ }^{16}$ zu nennen. Sie versuchten auf Grund von Altersbestimmungen nach der Uran/Blei-Methode und Messungen des Argongehaltes an Materialien, die nach geologischen Gesichtspunkten das gleiche Alter haben, das Verzweigungsverhältnis des $\mathrm{K}^{40} \mathrm{zu}$ berechnen. W a s s e r b u r g fand bei seinen Messungen einen Wert von $\lambda_{\mathrm{K}}: \lambda_{\beta}=0,085$. Das von uns gemessene Verzweigungsverhältnis ist trotz der vorhandenen Argonverluste um 20\% größer. Der Grund für die abweichenden Ergebnisse kann vielleicht eine unvollständige Argongewinnung bei dem von $\mathrm{W}$ a s s e r b u rg verwendeten $\mathrm{NaOH}$-Aufschluß sein.

Das von uns gefundene Verzweigungsverhältnis bestätigt die Vermutung, daß den direkten Messungen ${ }^{5-10}$ mehr Vertrauen entgegengebracht werden darf. Der Mittelwert der direkten Messungen beträgt nämlich: $\lambda_{\mathrm{K}}: \lambda_{\beta}=0,119 \pm 0,006$.

Wir setzen unsere Bemühungen dahin fort, ein wirklich einwandfreies Material zu bekommen, das möglichst kleine Argonverluste durch eine Kaolinisierung erlitten hat. Mit diesem Material hoffen wir, das von uns benutzte Verzweigungsverhältnis aus den direkten Messungen noch besser bestätigen $\mathrm{zu}$ können.

Die Deutsche Forschungsgemeinschaft unterstützte diese Untersuchungen mit einer Sachbeihilfe.

${ }^{14}$ G. J. W a s s e r b u r g u. R. J. H a y d e n, Phys. Rev. 93, 645 [1954]; 97, 86 [1955].

${ }_{15}$ G. J. W a s s e r b u rg u. R. J. H a y d e n, Geochim. cosmochim. Acta (London) 7, 51 [1955].

16 R. D. Russel, H. A. Shillibeer, R. M. F a q u h a r u. A. K. M o u s u f, Phys. Rev. 91, 1223 [1953]; 94, 1793 [1954]. 